\title{
G protein-coupled receptor, family C, group 5 (GPRC5B) downregulation in spinal cord neurons is involved in neuropathic pain
}

\author{
Hyung-Joo Chung ${ }^{1}$, Ju Deok Kim ${ }^{1}$, Kyung Han Kim ${ }^{1}$, and Na Young Jeong ${ }^{2}$ \\ ${ }^{1}$ Department of Anesthesiology and Pain Medicine, Kosin University College of Medicine, ${ }^{2}$ Department of Anatomy and Cell Biology \\ and Mitochondria Hub Regulation Center, Dong-A University College of Medicine, Busan, Korea
}

Background: G protein-coupled receptor, family C, group 5 (GPRC5B), a retinoic acid-inducible orphan G-proteincoupled receptor (GPCR), is a member of the group C metabotropic glutamate receptor family proteins presumably related in non-canonical Wnt signaling. In this study, we investigated altered GPRC5B expression in the dorsal horn of the spinal cord after spinal nerve injury and its involvement in the development of neuropathic pain.

Methods: After induction of anesthesia by intraperitoneal injection of pentobarbital ( $35 \mathrm{mg} / \mathrm{kg}$ ), the left L5 spinal nerve at the level of $2 \mathrm{~mm}$ distal to the L5 DRG was tightly ligated with silk and cut just distal to the ligature. Seven days after nerve injury, animals were perfused with $4 \%$ paraformaldehyde, and the spinal cords were extracted and post-fixed at $4^{\circ} \mathrm{C}$ overnight. To identify the expression of GPRC5B and analyze the involvement of GPRC5B in neuropathic pain, immunofluorescence was performed using several markers for neurons and glial cells in spinal cord tissue.

Results: After L5 spinal nerve ligation (SNL), the expression of GPRC5B was decreased in the ipsilateral part, as compared to the contralateral part, of the spinal dorsal horn. SNL induced the downregulation of GPRC5B in NeuN-positive neurons in the spinal dorsal horn. However, CNPase-positive oligodendrocytes, OX42-positive microglia, and GFAPpositive astrocytes were not immunolabeled with GPRC5B antibody in the spinal dorsal horn.

Conclusions: These results imply that L5 SNL-induced GPRC5B downregulation may affect microglial activation in the spinal dorsal horn and be involved in neuropathic pain. (Korean J Anesthesiol 2014; 66: 230-236)

Key Words: GPCR5B, Microglial activation, Neuroglial cell, Neuropathic pain, Spinal nerve injury.

\footnotetext{
Received: April 29, 2013. Revised: 1st, June 25, 2013; 2nd, August 7, 2013; 3rd, September 4, 2013; 4th, October 2, 2013. Accepted: October 22, 2013. Corresponding author: Na Young Jeong, M.D., Ph.D., Department of Anatomy and Cell Biology and Mitochondria Hub Regulation Center, Dong-A University College of Medicine, 3-1, Dongdaesin-dong, Seo-gu, Busan 602-714, Korea. Tel: 82-51-240-2752, Fax: 82-51-241-3767, E-mail: hyukpoka@naver.com

(c) This is an open-access article distributed under the terms of the Creative Commons Attribution Non-Commercial License (http:// creativecommons.org/licenses/by-nc/3.0/), which permits unrestricted non-commercial use, distribution, and reproduction in any medium, provided the original work is properly cited.
} 


\section{Introduction}

G-protein-coupled receptors (GPCRs) contain seven transmembrane domains and have a critical role in signal transduction; therefore, they are one of the most famous pharmaceutical targets [1]. GPCR, family C, group 5 (GPRC5B), alias retinoic acid-inducible gene 2 (Raig2), is a member of the Raig subfamily of type 3 (family C) GPCRs. These proteins have same homology with the metabotropic glutamate receptors (mGluRs) in their seven transmembrane domain regions [2,3]. Additionally, family $\mathrm{C}$, as well as the Raig and mGluR subfamilies, also contains $\mathrm{GABA}_{\mathrm{B}}$, calcium-sensing, and pheromone receptors, all of which have critical roles in neuronal function [4].

Along with GPRC5B, three other Raigs have also been discovered. Contrary to other family $\mathrm{C}$ members, the Raig receptors typically have short amino termini, like GPCR groups $A$ and B [1,3]. Lately, studies have provided evidence that the Raig receptors might have a role in Wnt signaling, even though ligands remain to be identified for any member of this orphan GPCR subfamily $[5,6]$.

Human GPRC5B mRNA expression is rich in the central nervous system [7]. Robbins et al. [8] unveiled that the molecular weight of GPRC5B was $68 \mathrm{kDa}$ by Western blot analysis. Additionally, immunohistochemical analysis of GPRC5B showed receptor expression in the brain and spinal cord [8].

However, GPRC5B expression alterations in the spinal cord after nerve injury and their influence on neuropathic pain remain to be reported. Therefore, we investigated the pattern of GPRC5B expression in the dorsal horn of the spinal cord after spinal nerve injury and its involvement in neuropathic pain.

\section{Materials and Methods}

\section{Animals and surgical procedure for inducing neuropathic pain}

All efforts were made to minimize animal suffering, to decrease the number of animals used, and to use alternatives to in vivo techniques. Ten-week-old male Wister rats (7 rats in each group) were used in this study. Animals were housed with a $12 \mathrm{~h}$ light/dark cycle (8:00/20:00) at a constant room temperature of $25^{\circ} \mathrm{C}\left(23 \pm 2^{\circ} \mathrm{C}\right)$ and humidity of $45-65 \%$. After induction of anesthesia by intraperitoneal injection of pentobarbital $(35 \mathrm{mg} / \mathrm{kg})$, the left L5 spinal nerve at the level of $2 \mathrm{~mm}$ distal to the L5 dorsal root ganglion was tightly ligated with silk and cut just distal to the ligature as previously described [9]. After this procedure, the wound was closed, and the rats were allowed to recover for 7 days. The procedures in this study were done according to protocols approved by the University Committee on Animal Research (KHUASP[SE]-13-027), which follows the guidelines for the use of experimental animals established by The Korean Academy of Medical Science.

\section{Behavioral test}

To screen for tactile allodynia-expressing animals, mechanical sensitivity was examined utilizing calibrated von Frey filaments $(0.4-15 \mathrm{~g})$ in a blinded manner. The $50 \%$ paw-withdrawal threshold (PWT) was determined by the up-down method [10]. Seven days after spinal nerve ligation (SNL), animals that showed a PWT threshold $(\mathrm{g})<5$ were sacrificed.

\section{Western blot analysis}

For Western blot analyses, cultured sciatic explants were prepared and thirty-five micrograms of total protein was separated and transferred to a nitrocellulose membrane (Amersham Bioscience, Piscataway, NJ, USA). The blots were reacted with the proper primary antibody (against GPRC5B or $\beta$-actin as control) and with horseradish peroxidase-conjugated secondary antibody. Detection was performed utilizing an enhanced chemiluminescence-Western blot system (Amersham Bioscience). LAS image-analysis system (Fujifilm, Tokyo, Japan) was used to quantification. All experiments were repeated a minimum of three times.

\section{Immunofluorescent staining}

Seven days after nerve injury, animals were perfused with $4 \%$ paraformaldehyde and $2.1 \%$ picric acid in $0.1 \mathrm{M}$ phosphate buffer (PB) for $30 \mathrm{~min}$, and the spinal cords were extracted and post-fixed at $4^{\circ} \mathrm{C}$ overnight. Then, a series of coronal sections of the spinal cord at the L5 level were cut with a microslicer at a thickness of $20 \mu \mathrm{m}$ and stored at $-80^{\circ} \mathrm{C}$ until used for immunofluorescent staining. Sections were incubated with primary antibodies against OX42, CNPase, GPRC5B, GFAP (Santa Cruz Biotechnology, Santa Cruz, CA, USA) or NeuN (Chemicon, Temecula, CA, USA) and with a fluorescent-conjugated secondary antibody (Alexa-488 or -594; Cell Signaling Technology). Fluorescence images were acquired utilizing a laser confocal microscope (LSM710; Carl Zeiss, Germany) and analyzed using Zeiss LSM Image Browser.

\section{Data analysis}

Data on immunostained sections from rats that had survived 7 days after the SNL $(n=5)$ were analyzed. Nine OX42-immunostained sections from the L5 layer were selected randomly from each rat. The numbers of OX42-positive microglia on the contralateral and ipsilateral sides were counted in a $6,000-\mu \mathrm{m}^{2}$ 
area within the L5 spinal cord. Differences in the means between groups were statistically assessed utilizing analysis of variance (ANOVA) followed by the Bonferroni post hoc test. Differences were considered statistically significant at $\mathrm{P}<0.01$. Error bars in the figures indicate the standard error of the mean.

\section{Results}

\section{L5 spinal nerve injury induces a decrease in GPRC5B expression in the ipsilateral dorsal horn}

GPRC5B is abundantly expressed in the spinal cord [8]. To determine changes in GPRC5B expression after SNL, we first performed a time course by Western blot analysis to measure changes in GPRC5B protein expression in the dorsal horn 7 days after L5 SNL. We found that GPRC5B expression was substantially decreased in the ipsilateral dorsal horn, but not in the contralateral part (Fig. 1). GPRC5B protein levels started to decrease in the ipsilateral dorsal horn at day 7 after SNL (Fig. 1).

To identify allodynia behavior in our experimental animals after SNL, we performed a behavior test. Mechanical allodynia was tested utilizing von Frey filaments. We found significant mechanical allodynia following SNL in our experimental animals

A

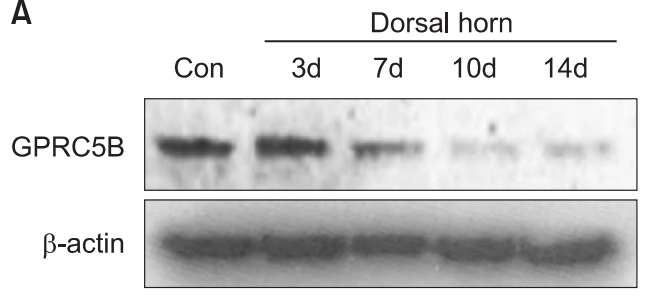

B

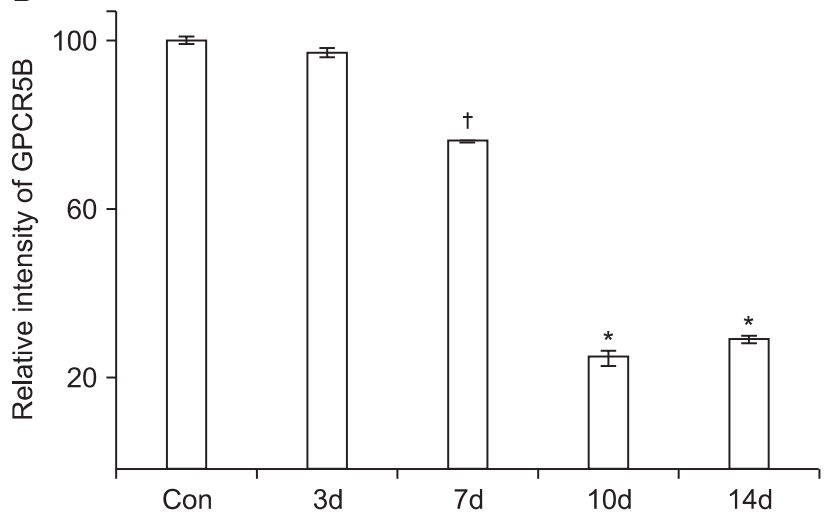

Fig. 1. GPRC5B protein expression decreases after SNL. (A) Decreased GPRC5B protein expression in the ipsilateral dorsal horn at day 7 after surgery as determined by Western blot analysis. (B) Densitometric analysis of Western blots. ${ }^{*} \mathrm{P}<0.01$ and ${ }^{\dagger} \mathrm{P}<0.05$ compared with the experimental control. GPRC5B: G protein-coupled receptor, family C, group 5, SNL: spinal nerve ligation, Con: Contralateral.
(Fig. 2). Thus, this functional assay supported the molecular and morphological evidence found in our experiments.

The changes in GPRC5B expression after spinal nerve injury were also confirmed by immunostaining (Fig. 3). GPRC5B immunofluorescence in the ipsilateral dorsal horn decreased after SNL compared with the contralateral part (Fig. 3). Microglia within the spinal dorsal horn are activated by a variety of nerve injuries such as compression, ligation, or transaction [11-13]. OX42 is an activated microglial marker that can indicate an injured spinal dorsal horn [11-13]. Thus, identification of microglial activation using OX42 suggests a morphological indication of pain induction. In the present study, we recognized that OX42 labeling was more outstanding in the dorsal horn ipsilateral to the nerve injury (Fig. 3). Thus, GPRC5B immunostaining was weaker in the dorsal horn on the side of nerve injury in comparison with the contralateral side, which showed normal OX42 immunoreactivity (Fig. 3).

\section{Downregulated expression of GPRC5B occurs in dorsal horn sensory neurons after spinal nerve injury}

To identify the cell types that showed changes in GPRC5B expression after L5 spinal nerve injury, we did double immunostaining of GPRC5B with several cell-specific markers: for oligodendrocytes, CNPase; for astrocytes, GFAP; for neurons, NeuN; and for microglia, OX42. Double immunofluorescence showed that GPRC5B colocalized with NeuN (Fig. 4B), but not with OX42 (Fig. 4A), GFAP (Fig. 4C), or CNPase (Fig. 4D), in both the ipsilateral and contralateral spinal dorsal horn after SNL. However, colocalization of GPRC5B and NeuN seemed relatively weaker in the ipsilateral part because of decreased GPRC5B expression (Fig. 4B). Therefore, GPRC5B was expressed in dor-

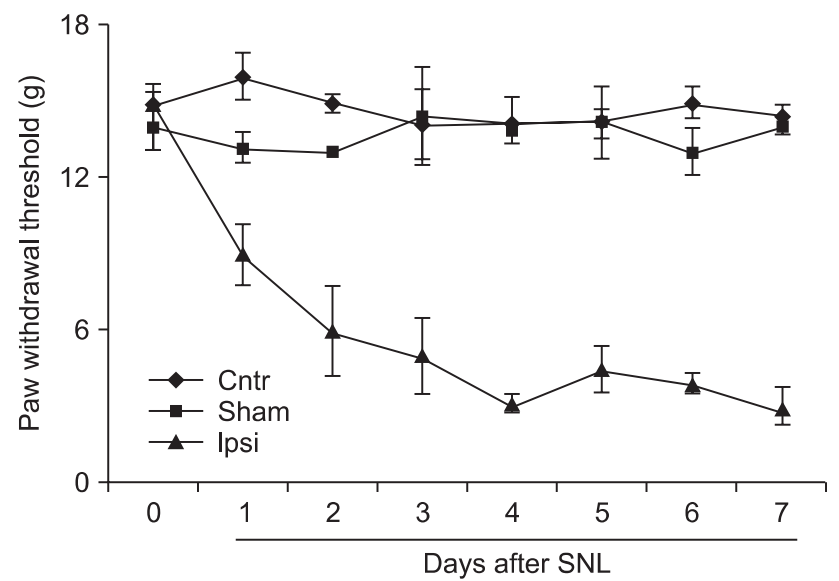

Fig. 2. Neuropathic pain behavior after SNL. The paw-withdrawal test (PWT) of the ipsilateral hindpaw in response to mechanical stimulation in rats was performed each day for 7 days after SNL. Cntr: contralateral, Sham: sham-control, Ipsi: ipsilateral. 
A
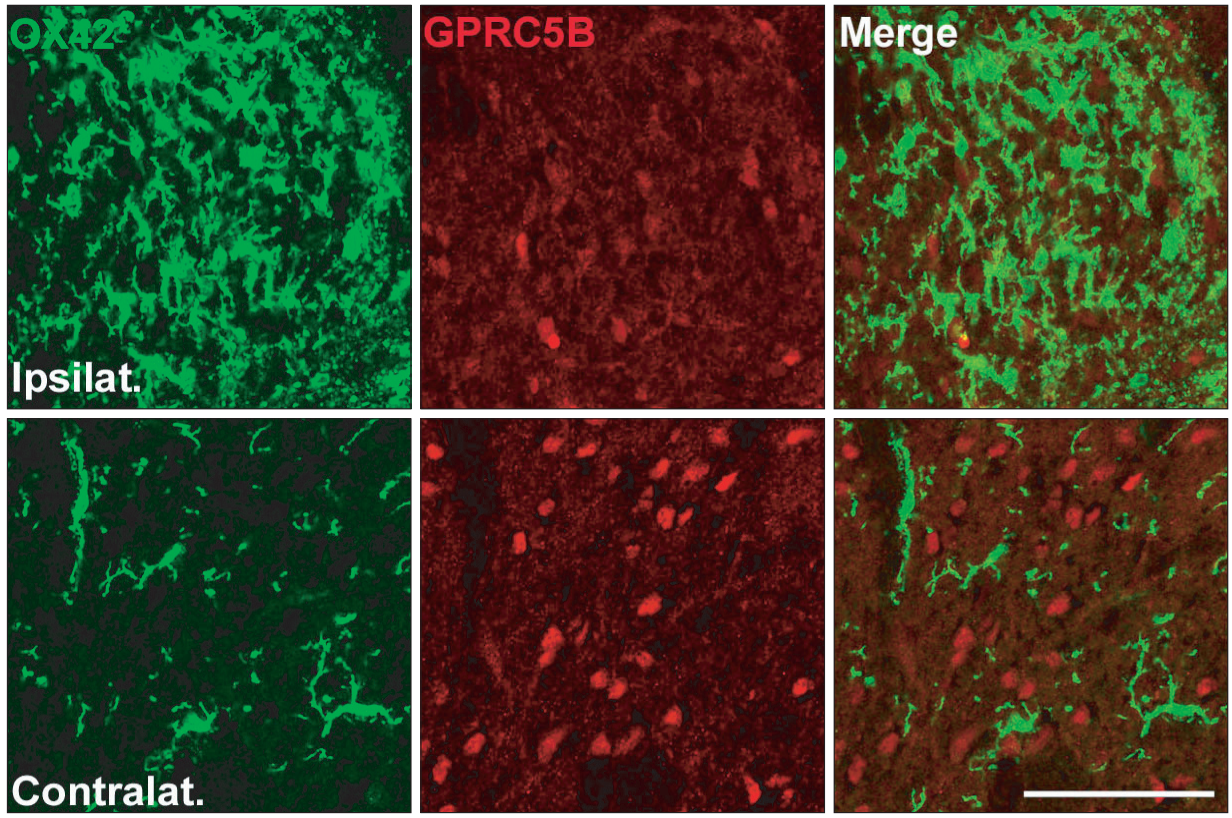

B

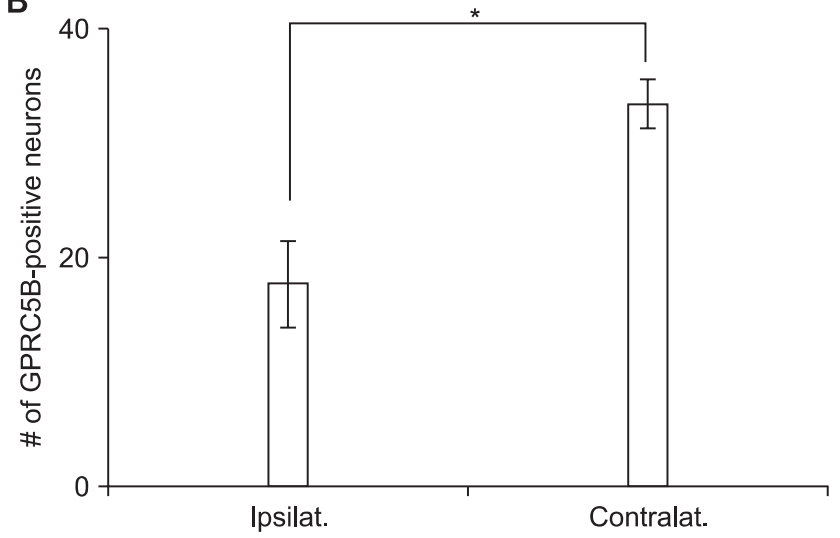

Fig. 3. GPRC5B immunofluorescence in the ipsilateral dorsal horn decreases after SNL. (A) Double staining of GPRC5B (red) and OX42 (green), a microglial marker. Two single-stained images were merged. Scale bar, $30 \mu \mathrm{m}$. (B) The number of GPRC5B-positive cells is shown in the graph. ${ }^{*} \mathrm{P}<0.01$ compared with the experimental control.

sal horn sensory neuron cells and downregulated after nerve injury.

\section{Discussion}

Pain is an unpleasant sensation that acts as a warning signal of imminent or actual injury. In this respect, pain is an important warning system that prevents damage to the body. However, chronic pain has no apparent value or physiological benefit but causes physical and emotional stress. Neuropathic pain is chronic pain resulting from an injury to the nervous system. Unfortunately, most neuropathic pain responds poorly to non-steroidal anti-inflammatory drugs and opiates. GPRCs represent more than $60 \%$ of drug targets and are major targets for hormones, neurotransmitters, and neuropeptides in the nervous system $[1,14]$. Therefore, GPRCs are important for drug development

targeting neuropathic pain.

In this study, we showed for the first time that after spinal nerve injury, the expression of GPRC5B, the newly identified GPCR that causes neuropathic pain after nerve injury, is downregulated in the spinal nerve dorsal horn. Here, we used the L5 SNL model of neuropathic pain to observe the morphological and molecular changes in the spinal dorsal horn after L4-5 spinal nerve injury [15]. As shown in Fig. 1, GPRC5B expression in the ipsilateral part relative to the nerve injury was decreased after SNL. This indicates that GPRC5B expressed in the spinal cord serves a biological function, but its action is decreased after nerve injury. We recognized that GPRC5B was expressed in the normal spinal cord and decreased after SNL only in neurons, but not in other neuroglial cells such as microglia, astrocytes, or oligodendrocytes, in the spinal dorsal horn (Fig. 4). It has been shown by many in vivo experiments that neuropathic pain 
A

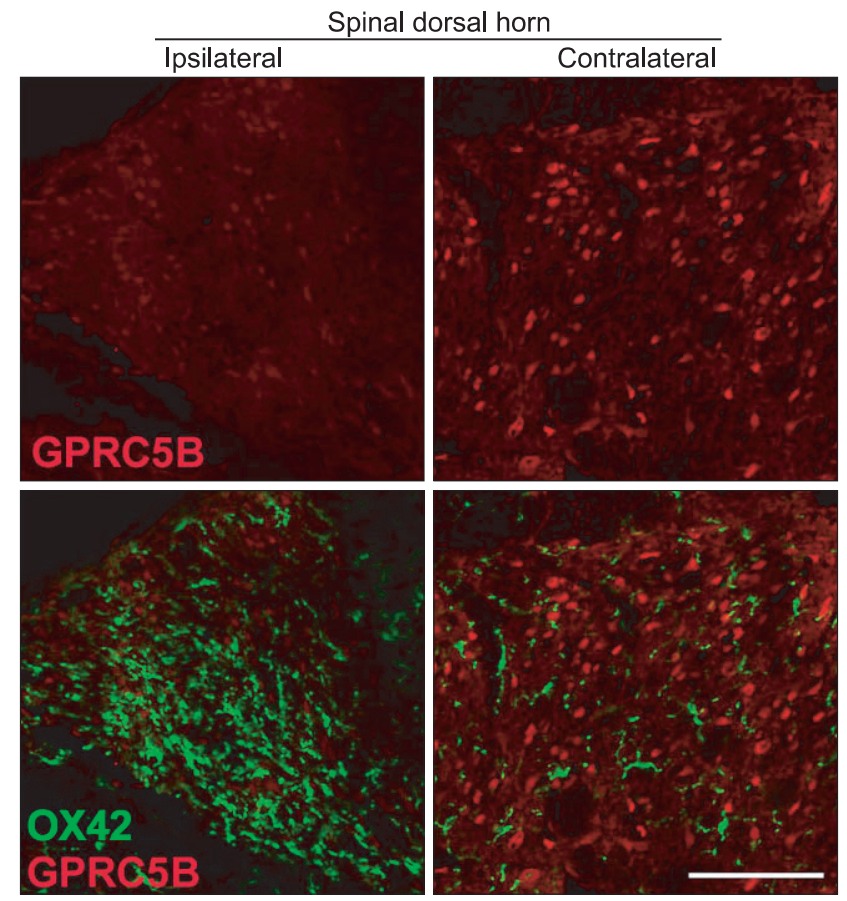

C
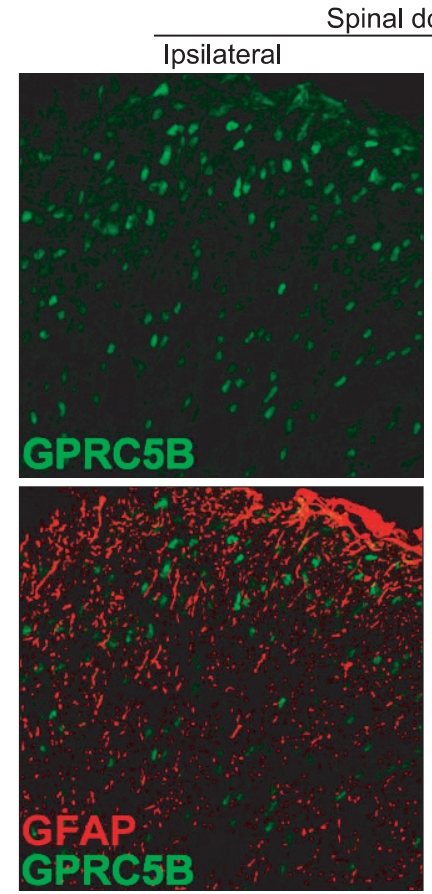

B

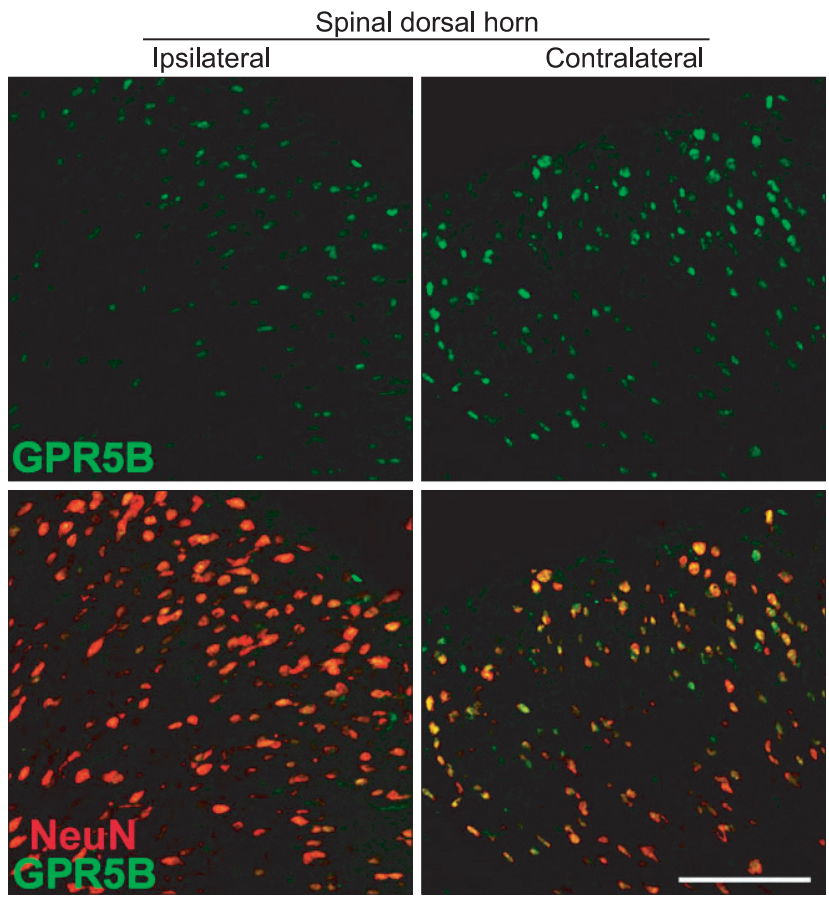

D

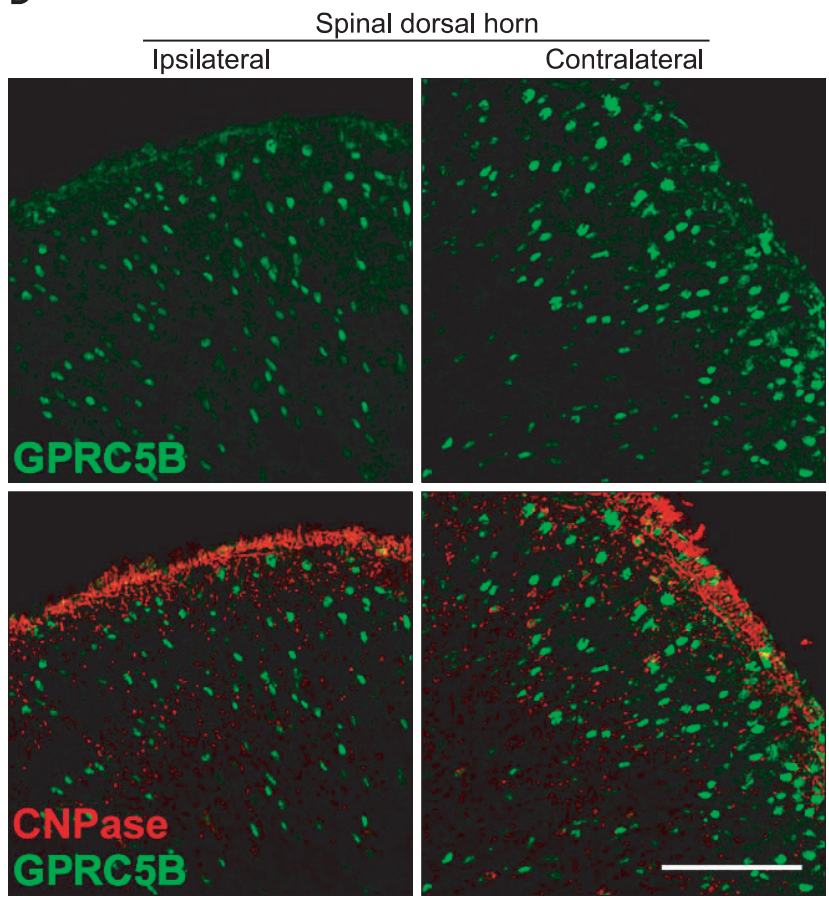

Fig. 4. L5 SNL induces GPRC5B downregulation in spinal neurons, but not in microglia. (A, B) GPRC5B colocalizes with NeuN (a neuron marker), but not with OX42 (a microglia marker), in both the ipsilateral and contralateral dorsal horn. Double-labeled cells appear yellow in the merged images. Scale bar, $100 \mu \mathrm{m}$. (C, D) Double staining of GPRC5B and GFAP (an astrocyte marker) or CNPase (an oligodendrocyte marker) showed no colocalization. Scale bar, $100 \mu \mathrm{m}$.

is caused by abnormal nerve excitability in primary sensory ganglia and the spinal dorsal horn after nerve injury. It is also reported that not only nerve cells but also microglial, astrocyte, and immune cells of the central nervous system cause neuropathic pain [16].

After peripheral nerve injury, microglia in the resting state 
in the spinal dorsal horn are converted to an activated state with a series of morphological and molecular changes. As activation progresses, microglia undergo progressive changes, including altered expression of cell-surface receptors, neurotransmitters, and diffusible factors. Astrocytes, a type of neuroglial cell, contribute to maintenance of neuropathic pain [17]. It has been suggested that microglia and astrocytes play important roles in the development and maintenance of nerve injury and neuropathic pain. Therefore, microglia and astrocytes are promising targets for inhibiting neuropathic pain and overexcited spinal dorsal horn neurons by pharmacologically and genetically modulating the molecular expression and function of, in particular, their cell-surface receptors. GPRC5B downregulation in spinal dorsal horn neurons might be related to microglial and astrocyte activation. First, given the apparent downregulation of GPRC5B in neurons after nerve injury, it is possible that GPRC5B downregulation may influence partial microglial activation directly or indirectly because of the significant decrease in GPR5B at the peak time of microglial activation, 7 days [11]. Additionally, our data suggest that GPRC5B downregulation may be involved in neuropathic pain, as evidenced by microglial activation in the ipsilateral side after nerve injury. Therefore, GPRC5B downregulation may affect the starting point of the development of neuropathic pain (Figs. 1 and 3) $[12,13,18]$. Second, the peak time of activation of astrocytes is later than that of microglia activation because astrocytes have a role in the maintenance of neuropathic pain [18]. Given that, in this study, we also observed significant downregulation of GPRC5B by Western blot in the ipsilateral spinal dorsal horn at the peak time of astrocyte activation, 10 days (Figs. 1C and 1D) [18], it seems likely that GPRC5B is associated with the induction and maintenance of neuropathic pain.

In the present study, we evaluated GPRC5B, one of the orphan GPCRs, to identify new GPRCs that induce neuropathic pain. The results showed that spinal nerve injury induces GPCR5B downregulation in the dorsal horn of the spinal cord. Furthermore, decreased GPRC5B expression may affect partial microglial activation as well as astrocyte activation in the spinal dorsal horn and may be involved in neuropathic pain.

Although the underlying mechanisms that regulate GPRC5B expression and neuropathic pain remain to be defined, further study of altered GPRC5B expression may offer promising strategies to establish the mechanisms of neuropathic pain and develop new drugs for controlling neuropathic pain.

\section{Acknowledgments}

This work was supported by the Dong-A University research fund.

\section{References}

1. Lagerström MC, Schiöth HB. Structural diversity of G protein-coupled receptors and significance for drug discovery. Nat Rev Drug Discov 2008; $7: 339-57$.

2. Bräuner-Osborne H, Krogsgaard-Larsen P. Sequence and expression pattern of a novel human orphan G-protein-coupled receptor, GPRC5B, a family C receptor with a short amino-terminal domain. Genomics 2000; 65: 121-8.

3. Robbins MJ, Michalovich D, Hill J, Calver AR, Medhurst AD, Gloger I, et al. Molecular cloning and characterization of two novel retinoic acid-inducible orphan G-protein-coupled receptors (GPRC5B and GPRC5C). Genomics 2000; 67: 8-18.

4. Strosberg AD. G protein coupled R7G receptors. Cancer Surv 1996; 27: 65-83.

5. Harada Y, Yokota C, Habas R, Slusarski DC, He X. Retinoic acidinducible G protein-coupled receptors bind to frizzled receptors and may activate non-canonical Wnt signaling. Biochem Biophys Res Commun 2007; 358: 968-75.

6. Kristiansen K. Molecular mechanisms of ligand binding, signaling, and regulation within the superfamily of G-protein-coupled receptors: molecular modeling and mutagenesis approaches to receptor structure and function. Pharmacol Ther 2004; 103: 21-80.

7. Robbins MJ, Michalovich D, Hill J, Calver AR, Medhurst AD, Gloger I, et al. Molecular cloning and characterization of two novel retinoic acid-inducible orphan G-protein-coupled receptors (GPRC5B and GPRC5C). Genomics 2000; 67: 8-18.

8. Robbins MJ, Charles KJ, Harrison DC, Pangalos MN. Localisation of the GPRC5B receptor in the rat brain and spinal cord. Brain Res Mol Brain Res 2002; 106: 136-44.

9. Tozaki-Saitoh H, Tsuda M, Miyata H, Ueda K, Kohsaka S, Inoue K. P2Y12 receptors in spinal microglia are required for neuropathic pain after peripheral nerve injury. J Neurosci 2008; 28: 4949-56.

10. Chaplan SR, Bach FW, Pogrel JW, Chung JM, Yaksh TL. Quantitative assessment of tactile allodynia in the rat paw. J Neurosci Methods 1994; 53: 55-63.

11. Tsuda M, Shigemoto-Mogami Y, Koizumi S, Mizokoshi A, Kohsaka S, Salter MW, et al. P2X4 receptors induced in spinal microglia gate tactile allodynia after nerve injury. Nature 2003; 424: 778-83.

12. Griffin RS, Costigan M, Brenner GJ, Ma CH, Scholz J, Moss A, et al. Complement induction in spinal cord microglia results in anaphylatoxin C5a-mediated pain hypersensitivity. J Neurosci 2007; 27: 8699-708. 
13. Maeda M, Tsuda M, Tozaki-Saitoh H, Inoue K, Kiyama H. Nerve injury-activated microglia engulf myelinated axons in a P2Y12 signalingdependent manner in the dorsal horn. Glia 2010; 58: 1838-46.

14. Congreve M, Langmead C, Marshall FH. The use of GPCR structures in drug design. Adv Pharmacol 2011; 62: 1-36.

15. Kim SH, Chung JM. An experimental model for peripheral neuropathy produced by segmental spinal nerve ligation in the rat. Pain 1992; 50: 355-63.

16. Tsuda M, Inoue K, Salter MW. Neuropathic pain and spinal microglia: a big problem from molecules in "small" glia. Trends Neurosci 2005; 28: 101-7.

17. Zhuang ZY, Gerner P, Woolf CJ, Ji RR. ERK is sequentially activated in neurons, microglia, and astrocytes by spinal nerve ligation and contributes to mechanical allodynia in this neuropathic pain model. Pain 2005; 114: 149-59.

18. Zhuang ZY, Wen YR, Zhang DR, Borsello T, Bonny C, Strichartz GR, et al. A peptide c-Jun N-terminal kinase (JNK) inhibitor blocks mechanical allodynia after spinal nerve ligation: respective roles of JNK activation in primary sensory neurons and spinal astrocytes for neuropathic pain development and maintenance. J Neurosci 2006; 26: 3551-60. 